\title{
Rigorous Solution to Plane Wave Scattering by an Arbitrary-Shaped Particle Embedded into a Cylindrical Cell of Similar Material
}

\author{
Constantine A. Valagiannopoulos \\ Microwaves and Fiber Optics Laboratory, School of Electrical and Computer Engineering, National Technical University of Athens \\ (NTUA), Heroon Polytechniou 9, Zografou, 15773 Athens, Greece \\ Correspondence should be addressed to Constantine A. Valagiannopoulos, valagiannopoulos@gmail.com \\ Received 10 May 2009; Accepted 8 July 2009 \\ Recommended by Charles Bunting \\ An infinite cylinder of arbitrary shape is embedded into a circular one, and the whole structure is illuminated by a plane wave. The \\ electromagnetic scattering problem is solved rigorously under the condition that the materials of the two cylinders possess similar \\ characteristics. The solution is based on a linear Taylor expansion of the scattering integral formula which can be useful in a variety \\ of different configurations. For the specific structure, its own far field response is given in the form of a double series incorporating \\ hypergeometric functions. The results are in good agreement with those obtained via eigenfunction expansion. Several numerical \\ examples concerning various shape patterns are examined and discussed.
}

Copyright ( $(2009$ Constantine A. Valagiannopoulos. This is an open access article distributed under the Creative Commons Attribution License, which permits unrestricted use, distribution, and reproduction in any medium, provided the original work is properly cited.

\section{Introduction}

The electromagnetic scattering by arbitrary-shaped formations is a very intriguing issue examined by many researchers with obvious applicability to microwave and optic frequencies. In [1], an inhomogeneous and arbitraryshaped scatterer is enclosed in a spherical cladding and scatters the incident field. The inclusion is treated as a nonspherical perturbation, and thus the equations of nonspherical potential are utilized, together with the wellknown vector spherical harmonics. Also in [2], the scattering response of a chiral cylinder of arbitrary cross section is obtained through the generalized multipole technique. The accuracy of the method is demonstrated by comparison with the eigenfunction expansion method.

In [3], the physical optics method is employed to solve the electromagnetic scattering by an inhomogeneous impedance cylinder with arbitrary bound. The induced electric and magnetic currents on the illuminated region are expressed by means of the inhomogeneous impedance boundary condition. Finally in [4], Tezel studies the case of an arbitrary-shaped object enclosed in a circular cylinder by taking the Taylor series expansion of fields with unknown coefficients on the circles that are inside and outside of object. The series equations are obtained through the boundary condition and by exploiting the orthogonality properties of Fourier expansion functions.

In this work, we examine a two-dimensional structure comprised of two layers, the outer of which has a circular bound, while the inner one possesses an arbitrary one. We obtain rigorously the solution to the plane wave scattering from this complex rod via the scattering integral under the simplifying assumption that the materials the two regions are filled with, are similar. In particular, we take the firstorder Taylor expansion of the scattering formula around the wavenumber of the external cell medium. The initial approximation of the total field is obtained as function of the incident one. The final result is expressed as an easily calculable double series whose terms incorporate hypergeometric functions. To validate the followed approach, we compare the results of the approximate method with the eigenfunction sums for a simple example that possesses analytic solution. To this end, various structures with cardioid-, astroid-, and egg-shaped bounds for the internal formation are studied, 


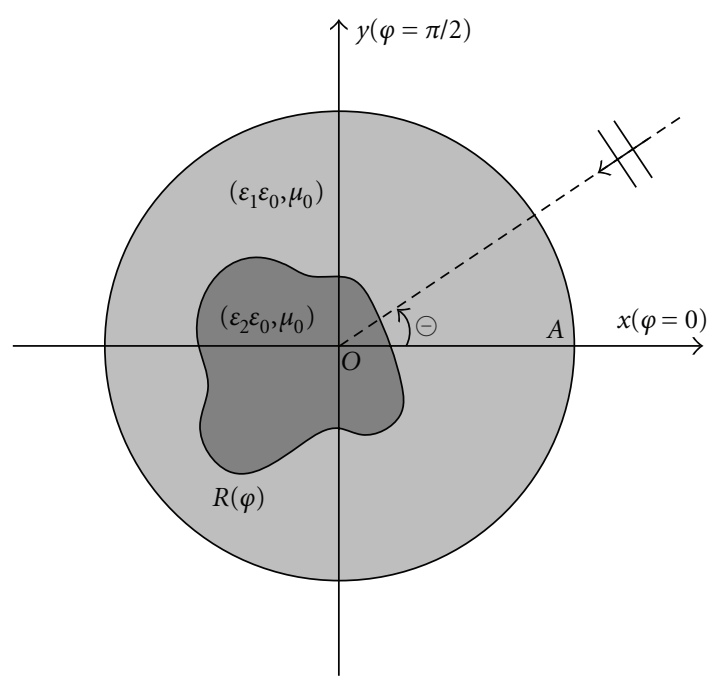

Figure 1: The physical configuration of the examined structure. A dielectric rod scatters the incident plane wave in the presence of an inclusion of arbitrary shape and similar texture.

and their far field response is represented as function of the incidence angle. The behavior of the curves is observed, and discussed and certain conclusions connecting the inclusion shapes with the variations are drawn and justified.

\section{Problem Definition}

We suppose the two-dimensional structure depicted in Figure 1 where the used cylindrical coordinate system $(\rho, \varphi, z)$ is also defined. An infinite cladding of circular cross section with radius $A$, made from a dielectric material with relative permittivity $\varepsilon_{1}$ (region 1 ), engulfs a particle of arbitrary shape and dielectric constant $\varepsilon_{2}$ (region 2 ). The formation is centralized at the origin $O$, and its bound is determined by the well-known polar formula $\rho=R(\varphi)$ with $0 \leq R(\varphi) \leq A$. The considered device is surrounded by the vacuum region 0 with intrinsic parameters $\left(\varepsilon_{0}, \mu_{0}\right)$, and it is excited by a $z$-polarized plane wave incident at angle $\Theta$ with respect to the horizontal axis, whose electric field possesses the following expansion [5, page 596]:

$$
E_{0, \text { inc }}^{\text {prim }}(\rho, \varphi)=\sum_{n=-\infty}^{+\infty}(-j)^{n} J_{n}\left(k_{0} \rho\right) e^{-j n(\varphi-\Theta)} .
$$

The notation $J_{x}(y)$ is used for the cylindrical Bessel function of order $x$ and argument $y$, while $k_{0}=\omega \sqrt{\varepsilon_{0} \mu_{0}}$ is the wavenumber into vacuum with a harmonic time dependence $e^{+j \omega t}$. The superscript prim is used to remark that this quantity is the primary component added to the secondary one $E_{0 \text {,inc }}^{\mathrm{sec}}(\rho, \varphi)$, expressing the reaction of the circular dielectric rod, to give the incident field into vacuum. Needless to say that the first subscript indicates the referred area, and therefore, the incident field into region 1 is comprised of a single component denoted by $E_{1 \text {,inc }}(\rho, \varphi)$. Both the shape and the excitation of the structure are invariant across the $z$ axis. Accordingly, the only nonzero electric component is the axial one $E=E_{z}$, and the problem is reduced to a scalar one. All the participating materials are taken magnetically inert. The purpose of this work is to find an analytic solution for the scattered field of the described configuration, despite the arbitrary cross section of the internal formation, with the reductive assumption that the characteristics of the two materials do not differ significantly, that is, $\varepsilon_{2} \cong \varepsilon_{1}[6]$.

\section{Mathematical Formulation}

One of the most useful formulas in electromagnetic theory is the scattering integral [7]. The scattered field, developed by a dielectric object, is defined as an integral of Green's function multiplied by the unknown total field, over the volume of this inhomogeneity. If one denotes the wavenumbers inside the dielectric object and the host material with $k_{2}$ and $k_{1}$, respectively, the scattering integral in examining a twodimensional case is particularized to give [8]:

$$
E_{T}\left(k_{1}, k_{2}\right)=E_{I}\left(k_{1}\right)+\left(k_{2}^{2}-k_{1}^{2}\right) \iint_{(S)} G\left(k_{1}\right) E_{T}\left(k_{1}, k_{2}\right) d s .
$$

Mind that the dependencies on the geometrical parameters are suppressed and the volume integral has been replaced by a double integral on the cross section $(S)$ of the scatterer. The notations $G\left(k_{1}\right), E_{I}\left(k_{1}\right)$ to Green's function and the incident field which are computed in the absence of the inhomogeneity and thus are not related with its material $k_{2}$. The total electric field throughout the configuration is denoted by $E_{T}\left(k_{1}, k_{2}\right)$.

By considering the Taylor expansion of the function $E_{T}\left(k_{1}, k_{2}\right)$ with respect to $k_{2}$ for values close to $k_{1}$, one obtains the following simplified equation:

$$
E_{T}\left(k_{1}, k_{2}\right) \cong E_{I}\left(k_{1}\right)+2 k_{1}\left(k_{2}-k_{1}\right) \iint_{(S)} G\left(k_{1}\right) E_{I}\left(k_{1}\right) d s .
$$

In this sense, the unknown electric field inside the scatterer (which equals the total field in this area) is computed by $E_{T}\left(k_{1}, k_{2}\right) \cong\left(2 k_{1} /\left(k_{1}+k_{2}\right)\right) E_{I}\left(k_{1}\right)$, which is a more sophisticated approximation than the classical Born formula [9]: $E_{T}\left(k_{1}, k_{2}\right) \cong E_{I}\left(k_{1}\right)$, as it takes into account the texture of the scatterer. In particular, it covers the case of a conducting object whose field is inversely related to the material's conductivity and implicitly with the magnitude $\left|k_{2}\right|$.

The aforementioned analysis is a general purpose one as it can cover any two-dimensional problem. If one wishes to use it in studying the considered device, one can define the corresponding case-oriented quantities. Green's function $G\left(k_{1}\right)$ equals to the axial electric field of current $j / \omega \mu_{0}$ Amperes produced by a dipole source positioned along the axis $(\rho=P, \varphi=\Phi)$ at the observation point $(\rho, \varphi)$. It is noted that $P<A$ (as the inhomogeneity is contained into region 1) and $\rho>A$ (as we are interested to the far field of the device into region 0$)$. The analytic expression of $G(\rho, \varphi, P, \Phi)$ 
(where the geometrical variables are appeared) is given by [10]

$$
G(\rho, \varphi, P, \Phi)=\sum_{n=0}^{+\infty} C_{G}(n) H_{n}^{(2)}\left(k_{0} \rho\right) J_{n}\left(k_{1} P\right) \cos [n(\varphi-\Phi)]
$$

with

$$
C_{G}(n)=\frac{1}{2 \pi A} \cdot \frac{2-\delta_{n 0}}{k_{1} J_{n}^{\prime}\left(k_{1} A\right) H_{n}^{(2)}\left(k_{0} A\right)-k_{0} J_{n}\left(k_{1} A\right) H_{n}^{(2)^{\prime}}\left(k_{0} A\right)} .
$$

The symbol $\delta_{n m}$ is used for Kronecker's delta, while $H_{x}^{(2)}(y)$ is the cylindrical second type Hankel function of order $x$ and argument $y$.

Given the fact that the integral in (3) extends over the region 2 , the quantity $E_{I}\left(k_{1}\right)$ corresponds to the field $E_{1, \text { inc }}(P, \Phi)$ defined through

$$
E_{1, \text { inc }}(P, \Phi)=\sum_{m=0}^{+\infty} C_{1 I}(m) J_{m}\left(k_{1} P\right) \sin [m(\Phi-\Theta)]
$$

with

$$
C_{1 I}(m)=\frac{2 j}{\pi A} \cdot \frac{\left(2-\delta_{m 0}\right)(-j)^{m}}{k_{1} J_{m}^{\prime}\left(k_{1} A\right) H_{m}^{(2)}\left(k_{0} A\right)-k_{0} J_{m}\left(k_{1} A\right) H_{m}^{(2)^{\prime}}\left(k_{0} A\right)} .
$$

The field $E_{I}\left(k_{1}\right)$ in (3) outside the integral concerns the points with $\rho>A$ and equals to

$$
E_{0, \text { inc }}^{\mathrm{sec}}(\rho, \varphi)=\sum_{n=0}^{+\infty} C_{0 I}(n) H_{n}^{(2)}\left(k_{0} \rho\right) \cos [n(\varphi-\Theta)]
$$

with

$$
\begin{aligned}
C_{0 I}(n)= & \left(2-\delta_{n 0}\right)(-j)^{n} \\
& \cdot \frac{k_{0} J_{n}\left(k_{1} A\right) J_{n}^{\prime}\left(k_{0} A\right)-k_{1} J_{n}^{\prime}\left(k_{1} A\right) J_{n}\left(k_{0} A\right)}{k_{1} J_{n}^{\prime}\left(k_{1} A\right) H_{n}^{(2)}\left(k_{0} A\right)-k_{0} J_{n}\left(k_{1} A\right) H_{n}^{(2) \prime}\left(k_{0} A\right)} .
\end{aligned}
$$

The relations (5a)-(6b) are well known and have been carefully derived in $[8,10]$. The total electric field $E_{T}\left(k_{1}, k_{2}\right)$ of (3) is referred as $E_{0}(\rho, \varphi)$, and we are mainly interested in the scattered field developed by the internal formation $E_{0, \text { scat }}(\rho, \varphi)=E_{0}(\rho, \varphi)-E_{0 \text {,inc }}^{\text {prim }}(\rho, \varphi)-E_{0 \text {,inc }}^{\text {sec }}(\rho, \varphi)$, for an observer located into region 1 .

The double integral over the cross section of the arbitrary scatterer is written in polar coordinates [11] and can be

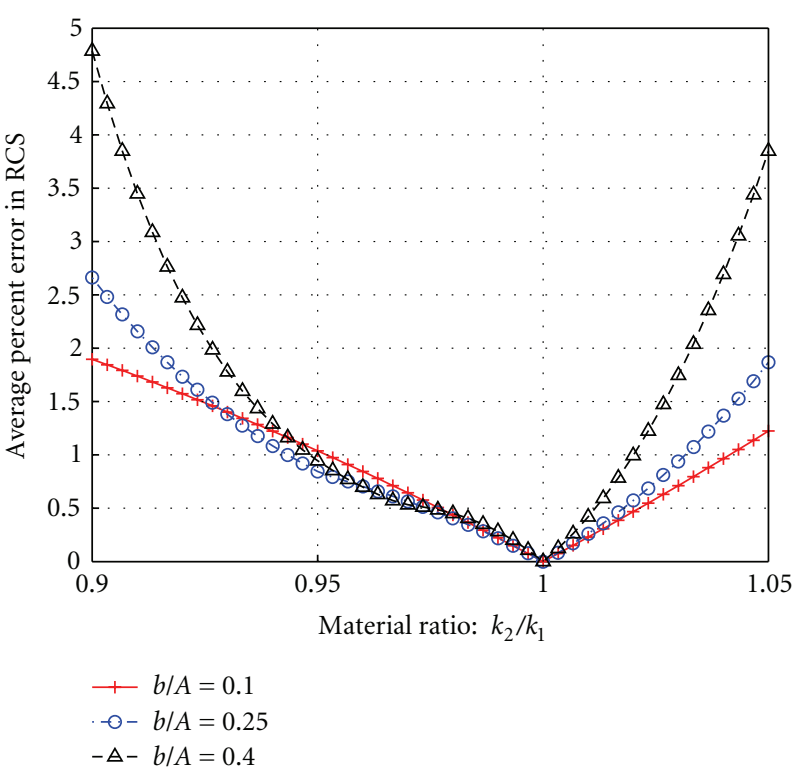

FIGURE 2: The average percent error of the method in computing $\mathrm{RCS}$, as function of the wavenumber ratio for various sizes of the concentric cylinder. Plot parameters are $k_{0}=2 \pi / \lambda_{0}=2000 \pi, k_{1}=$ $3 k_{0}, A=0.5 \lambda_{0}, \Theta=0$.

analytically evaluated through nontrivial integrals of Bessel functions [12] as follows:

$$
\begin{aligned}
& E_{0, \text { scat }}(\rho, \varphi) \\
& =2 k_{1}\left(k_{2}-k_{1}\right) \iint_{(S)} G\left(k_{1}\right) E_{I}\left(k_{1}\right) d s \\
& =2 k_{1}\left(k_{2}-k_{1}\right) \sum_{n=0}^{+\infty} \sum_{m=0}^{+\infty} C_{G}(n) C_{I 1}(m) H_{n}^{(2)}\left(k_{0} \rho\right) \\
& \quad \times \int_{0}^{2 \pi} \cos [n(\varphi-\Phi)] \cos [m(\Phi-\Theta)] \Psi(n, m, \Phi) d \Phi
\end{aligned}
$$

where the auxiliary function $\Psi(n, m, \Phi)$ is defined via

$$
\begin{aligned}
\Psi(n, m, \Phi)= & \int_{0}^{R(\Phi)} J_{n}\left(k_{1} P\right) J_{m}\left(k_{1} P\right) P d P \\
= & {\left[\frac{k_{1} R(\Phi)}{2}\right]^{n+m} } \\
& \times \frac{F\left(\mathfrak{W}, \mathfrak{F},-k_{1}^{2} R^{2}(\Phi)\right)}{(n+m+2) n ! m !} \\
& \times R^{2}(\Phi) .
\end{aligned}
$$

where $\mathfrak{W}$ denotes $[(n+m+1) / 2,(n+m+2) / 2,(n+m+2) / 2]$ and $\mathfrak{F}$ denotes $[m+1,(n+m+4) / 2, n+1, n+m+1]$. The notation $F(\mathbf{u}, \mathbf{v}, z)$ corresponds to the hypergeometric function with input vectors $(\mathbf{u}, \mathbf{v})$ and $\operatorname{argument} z$. 


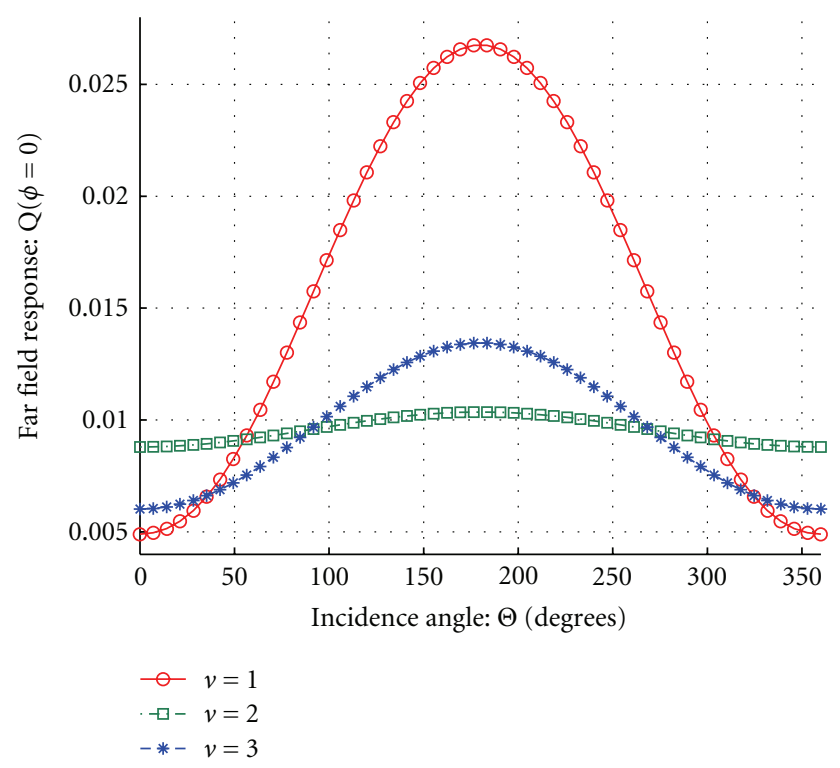

(a)

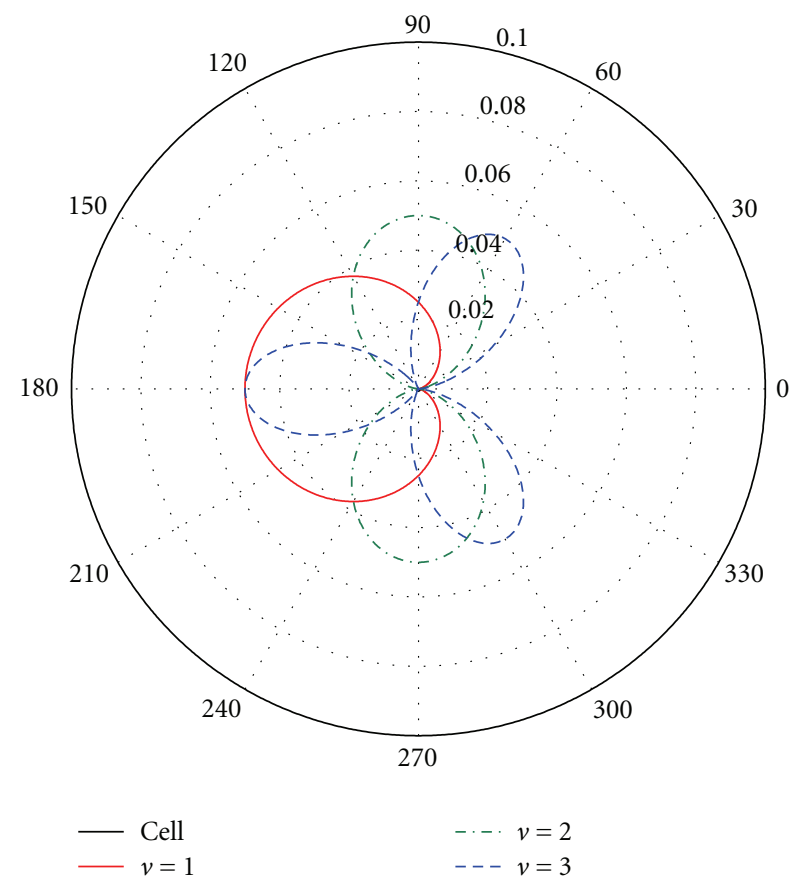

(b)

Figure 3: (a) The far field response along the horizontal direction, as function of the incidence angle for various cardioid shapes. (b) The polar plots of the corresponding cardioid shapes. Plot parameters are $k_{0}=2 \pi / \lambda_{0}=2000 \pi, k_{1}=3 k_{0}, k_{2}=1.05 k_{1}, A=$ $0.1 \lambda_{0}, a=0.5 \mathrm{~A}$.

\section{Numerical Results}

A set of computer programs has been developed to implement the proposed technique for a variety of internal formations. Prior to presenting the results, we should validate the aforementioned analysis when applied to a simple example possessing analytic solution. In particular, we consider a concentric circular core of radius $b<A$ into the external rod, and we compute the radar cross section (RCS) $\sigma(\varphi)$ via two alternative ways: (i) canonical solution and (ii) described method for $R(\Phi)=b$. The RCS in two dimensions is defined by $[5$, page 578$]$

$$
\sigma(\varphi)=\lim _{\rho \rightarrow+\infty}\left[2 \pi \rho\left|\frac{E_{0, \text { inc }}^{\mathrm{sec}}(\rho, \varphi)+E_{0, \text { scat }}(\rho, \varphi)}{E_{0, \text { inc }}^{\text {prim }}(\rho, \varphi)}\right|^{2}\right],
$$

where the Hankel functions are replaced by their asymptotic expressions for large arguments [13]:

$$
H_{n}^{(2)}\left(k_{0} \rho\right) \sim \sqrt{\frac{2}{\pi k_{0} \rho}} e^{-j\left(k_{0} \rho-(\pi / 4)\right)} j^{n}, \quad \rho \rightarrow+\infty .
$$

The double series in (7a) needs only a few terms to converge (for not electrically large scatterers) due to the two factorials in the denominator of (7b). Also, the azimuthal integration in (7a) is performed numerically with no problems-it concerns smooth integrand functions.

In Figure 2, the percent error of the method in computing RCS, averaged for $\varphi \in(0,2 \pi)$, is represented as function of the ratio $k_{2} / k_{1}$ for various radii $b$. Obviously, the error is nullified for $k_{2}=k_{1}$, that is, the case of a homogeneous cylindrical rod, where the term $E_{0 \text {,scat }}(\rho, \varphi)$ equals to zero. The method's error is increasing with increasing degree of inhomogeneity and with increasing size of the inclusion. However, within the range $0.93<k_{2} / k_{1}<1$, the recorded error does not vary substantially with $b$. Note that the difference is kept overall below 5\%, and therefore the results of the described technique are satisfactorily accurate when a ratio $k_{2} / k_{1}$ is close to unity as in the following examples.

In all the numerical simulations, we chose to examine the dependencies of the quantity defined as follows:

$$
Q(\varphi)=\lim _{\rho \rightarrow+\infty} \frac{2 \pi\left|E_{0, \text { scat }}(\rho, \varphi)\right|}{\int_{0}^{2 \pi}\left|E_{0, \text { inc }}^{\sec }(\rho, \varphi)\right| d \varphi} .
$$

The function $Q(\varphi)$ expresses the normalized effect of the arbitrary-shaped formation on the far field pattern of the cylindrical column. As the incidence angle $\Theta$ is a variable in the attached diagrams, it suffices to evaluate the far field response $Q(\varphi)$ along the horizontal axis $\varphi=0$. In Figure 3(a) we present the variation of $Q(\varphi=0)$ with respect to $\Theta$ for various cardioid-shape bounds of the inner particle appearing in Figure 3(b). The polar equations of the cardioid curves family are given by

$$
R_{\text {cardioid }}(\Phi, v, a)=\frac{a}{2}[1-\cos (\nu \Phi)], \quad \nu \in \mathbb{N}^{*}
$$

Regardless of the shape, the response is maximized at $\Theta=$ $180^{\circ}$ as the source producing the plane wave is located opposite to the observation point, and thus we receive a direct ray. The larger output is recorded for $v=1$ which means that the presence of the inner scatterer along 


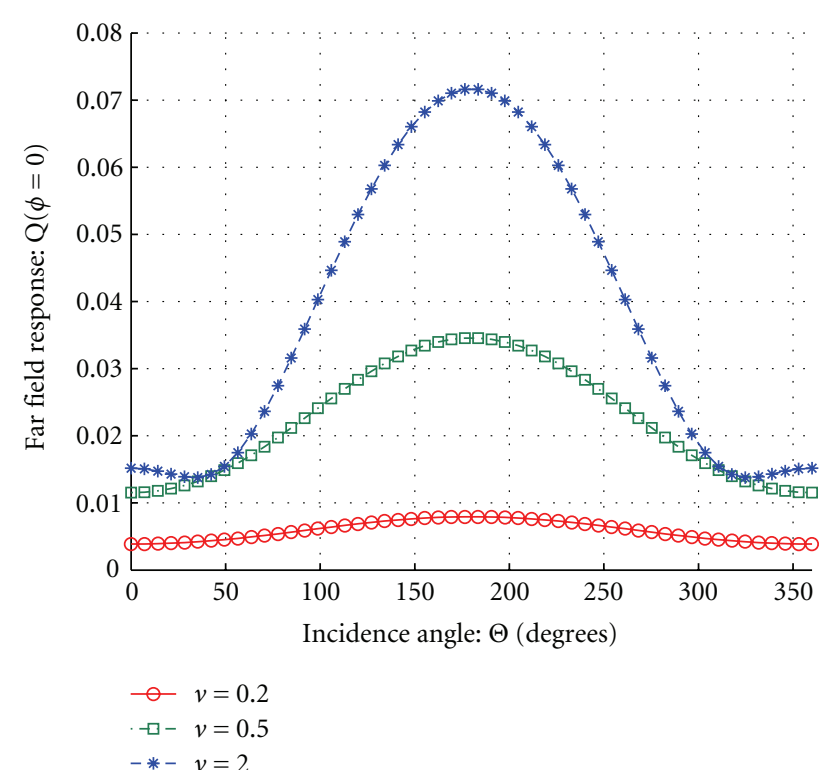

(a)

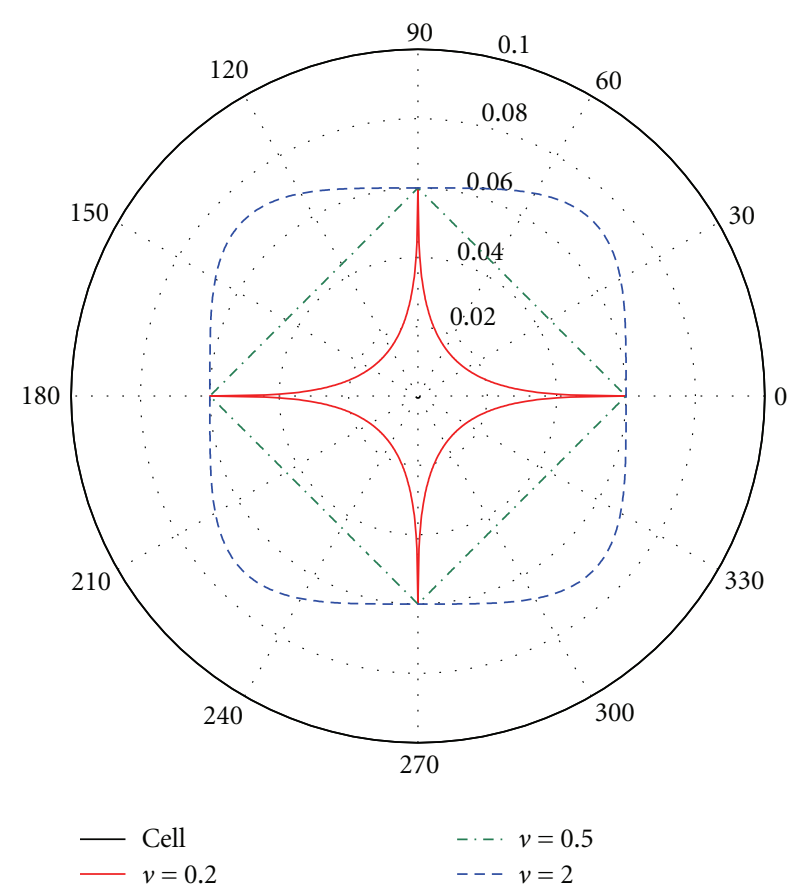

(b)

FIgURE 4: (a) The far field response along the horizontal direction, as function of the incidence angle for various astroid shapes. (b) The polar plots of the corresponding astroid shapes. Plot parameters are $k_{0}=2 \pi / \lambda_{0}=2000 \pi, k_{1}=3 k_{0}, k_{2}=1.05 k_{1}, A=0.1 \lambda_{0}, a=$ $0.6 A$.

the horizontal axis plays an amplifying role. It should be remarked that the higher the maximum is, the larger the fluctuation of the curve is; in other words, the received field at $\varphi=0$ is much less dependent on $\Theta$ for $\nu=2$ than in case of $v=1$. In addition, the minimum response is exhibited in all cases at $\Theta=0$, because the formations are not dense enough to reflect the incident wave.

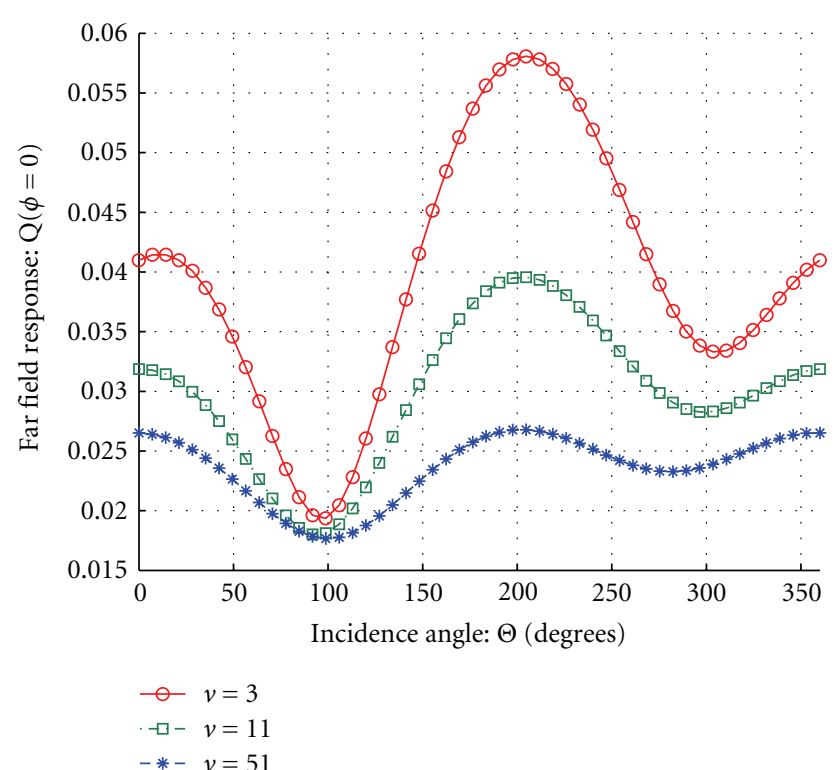

(a)

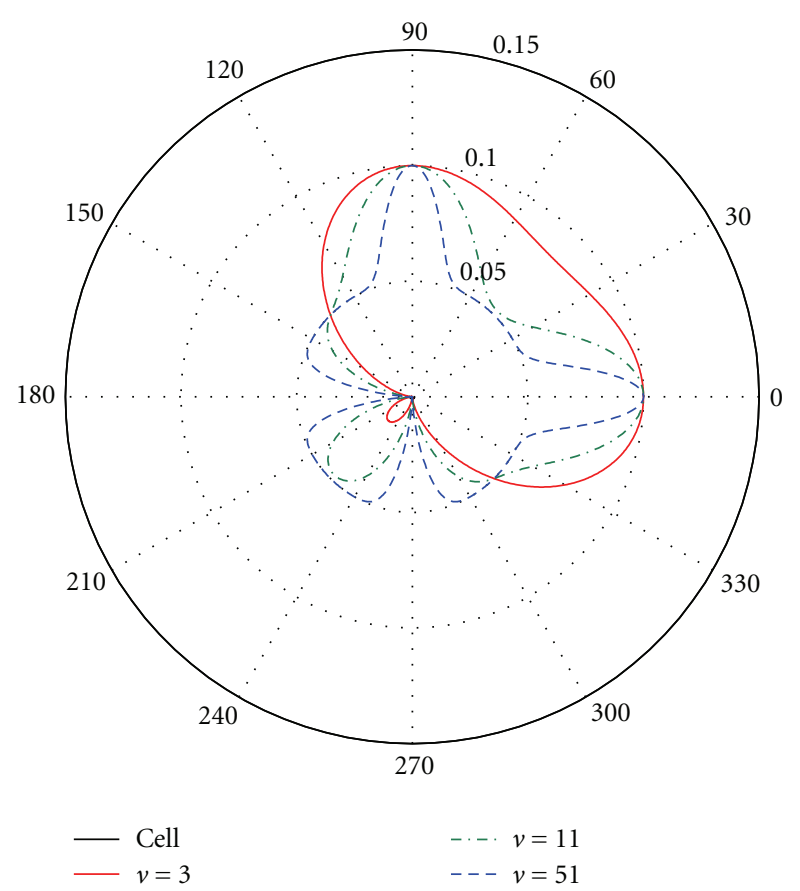

(b)

Figure 5: (a) The far field response along the horizontal direction, as function of the incidence angle for various egg shapes. (b) The polar plots of the corresponding egg shapes. Plot parameters: $k_{0}=$ $2 \pi / \lambda_{0}=2000 \pi, k_{1}=3 k_{0}, k_{2}=1.05 k_{1}, A=0.15 \lambda_{0}, a=0.33 A$.

In Figure 4(a) we present the variation of $Q(\varphi=0)$ with respect to $\Theta$ for various astroid-shape bounds of the inner particle appearing in Figure 4(b). The polar equations of the astroid curves family are given by

$$
R_{\text {astroid }}(\Phi, v, a)=a\left[\sin ^{2 v} \Phi+\cos ^{2 v} \Phi\right]^{-1 / 2 v}, \quad v \in(0,+\infty)
$$


Mind the symmetry of the curves reflecting the symmetry of the internal formation with respect to the horizontal axis. One can observe that the far field response is proportional to the size of the scatterer and thus is naturally maximized for the case of $v=2$. The negligible received field from the scatterer with $v=0.2$, despite its sharp edges (shown in Figure 4(b)), is clearly owed to the penetrable material they are constructed of; the results would be very different in case of a perfectly conducting core of the same shape. Furthermore, the curves for $v=0.5$ and $v=2$ coincide for $\Theta=45^{\circ}, 315^{\circ}$ where the latter one exhibits a local minimum due to the large front of the corresponding scatterer (otherwise the minimum would be recorded at $\Theta=$ 0 as happens in the cases of $v=0.2,0.5$ ).

In Figure 5(a) we present the variation of $Q(\varphi=0)$ with respect to $\Theta$ for various egg-shape bounds of the inner particle appearing in Figure 5(b). The polar equations of the egg curves family are given by

$$
R_{\mathrm{egg}}(\Phi, v, a)=a\left[1+\sin ^{\nu} \Phi+\cos ^{\nu} \Phi\right], \quad \nu \in \mathbb{N}^{*}
$$

Now the diagrams are not symmetric with respect to a value of $\Theta$, which is dictated by the corresponding asymmetry of the polar plots in Figure 5(b). The values of $Q(\varphi=0)$ are again small as happens in all the previous figures due to the tiny deviation of the wavenumber ratio $k_{2} / k_{1}$ from unity. There is an almost common minimum at $\Theta=100^{\circ}$ where the wave is reflected towards $\varphi=0$ in the same way for each of the three different boundaries of Figure 5(b). The distance between the curves gets substantial at $\Theta=200^{\circ}$, to close again to a moderate extent at $\Theta=300^{\circ}$.

\section{References}

[1] P. Rennert, "The mie scattering at an inhomogeneous and arbitrary shaped inclusion," Annalen Der Physik, vol. 502, pp. 27-40, 1989.

[2] M. Zhang and W. X. Zhang, "Scattering of electromagnetic waves from a chiral cylinder of arbitrary cross section-GMT approach," Microwave and Optical Technology Letters, vol. 10, no. 1 , pp. 22-25, 1995.

[3] N. S. Tezel and S. Paker, "Electromagnetic scattering by arbitrary shaped inhomogeneous impedance cylinder using physical optics method," Microwave and Optical Technology Letters, vol. 48, no. 7, pp. 1282-1286, 2006.

[4] N. S. Tezel, "A new formulation and solution method of electromagnetic scattering from arbitrary shaped dielectric objects," Microwave and Optical Technology Letters, vol. 50, no. 11, pp. 2768-2772, 2008.

[5] C. A. Balanis, Advanced Engineering Electromagnetics, John Wiley \& Sons, New York, NY, USA, 1989.

[6] C. A. Valagiannopoulos, "Electromagnetic scattering from two eccentric metamaterial cylinders with frequency-dependent permittivities differing slightly each other," Progress in Electromagnetic Research B, vol. 3, pp. 23-34, 208.

[7] C. T. Tai, Dyadic Green Functions in Electromagnetic Theory, IEEE Press, New York, NY, USA, 1994.

[8] C. A. Valagiannopoulos, "Arbitrary currents on circular cylinder with inhomogeneous cladding and RCS optimization," Journal of Electromagnetic Waves and Applications, vol. 21, no. 5, pp. 665-680, 2007.
[9] J. A. Hudson and J. R. Heritage, "The use of the born approximation in seismic scattering problems," International Geophysical Journal, vol. 66, no. 1, pp. 221-240, 1981.

[10] C. A. Valagiannopoulos, "On examining the influence of a thin dielectric strip posed across the diameter of a penetrable radiating cylinder," Progress in Electromagnetic Research C, vol. 3, pp. 203-214, 2008.

[11] W. Kaplan, Advanced Calculus, Publishing House of Electronic Industry, 2003.

[12] Y. L. Luke, Integrals of Bessel Functions, McGraw-Hill, New York, NY, USA, 1962.

[13] M. Abramowitz and I. Stegun, Handbook of Mathematical Functions, National Bureau of Standards, 1964. 

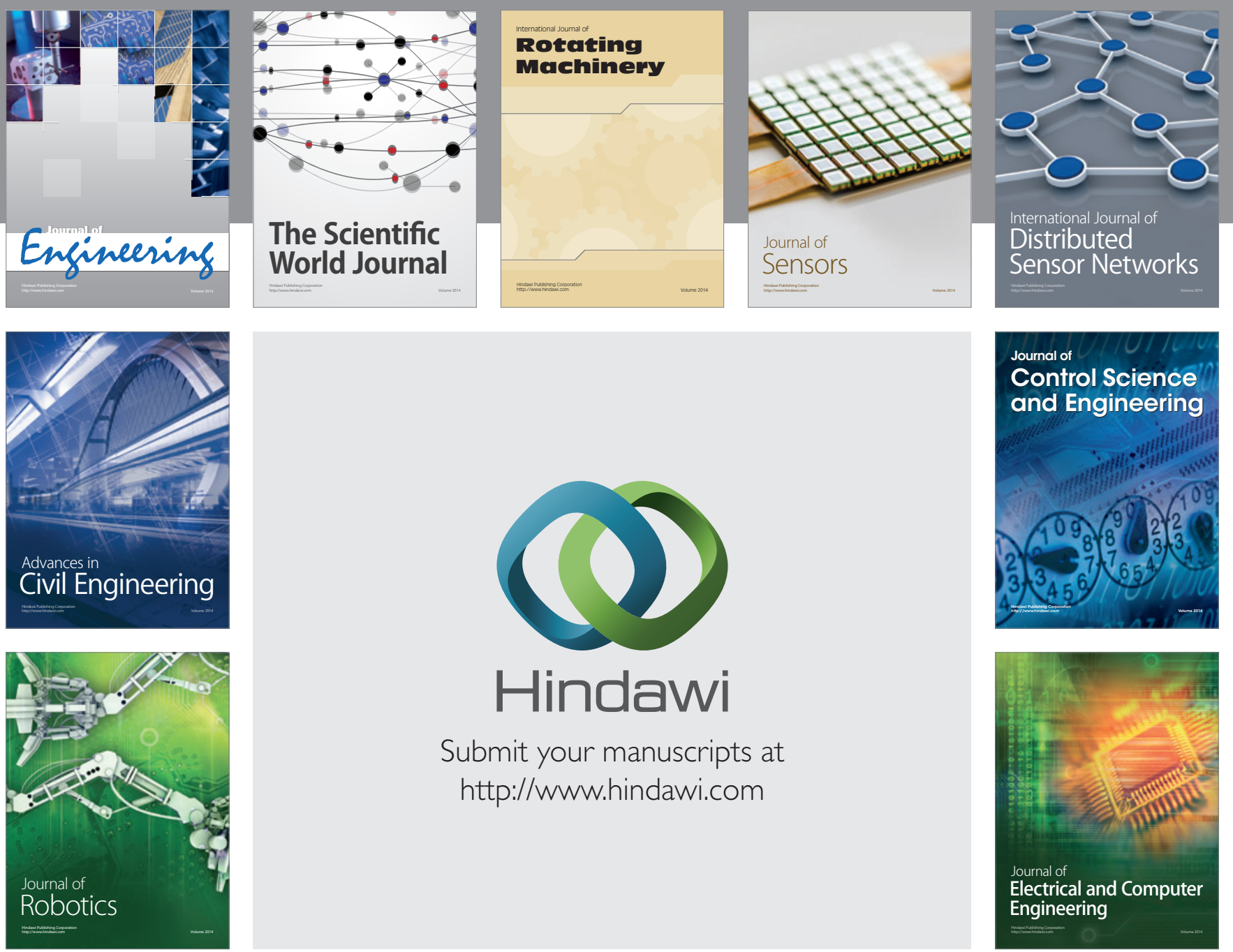

Submit your manuscripts at

http://www.hindawi.com
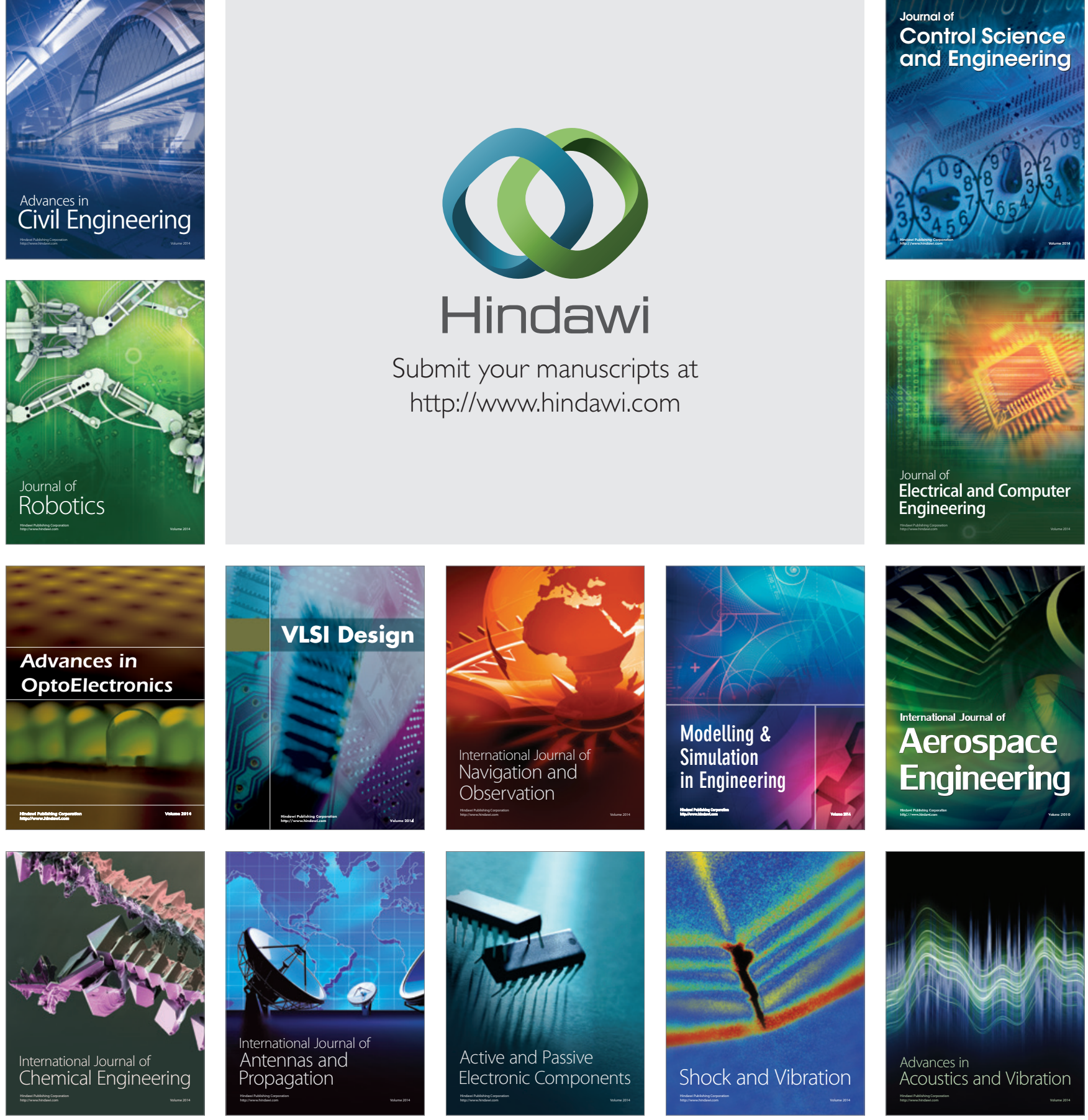